INVESTIGACIONES

\title{
¿Qué debe saber y saber hacer un profesor de estudiantes con talento académico? Una propuesta de estándares de formación inicial en educación de talentos
}

\author{
What should a teacher of talented students know and know how to do? \\ A proposal for initial training standards in talents' education
}

O que um professor de estudantes com aptidão para a aprendizagem deve conhecer e saber fazer? Uma proposta de critérios centrais a serem considerados na formação inicial de professores desses estudantes

\section{Piedad Cabrera*}

Centro de Estudios y Desarrollo de Talentos-PENTA UC. Pontificia Universidad Católica de Chile. Dirección Postal: Avenida El Bosque 565 dpto. 306. Número celular 77099024, epcabrer@uc.cl.

\begin{abstract}
RESUMEN
Se presentan los resultados de una investigación cualitativa, cuyo propósito fue generar una propuesta de estándares de formación de profesional docente en educación de talentos. Cuatro fases implementadas permitieron obtener los 'saberes' y 'saberes hacer' a considerar en la formación de profesionales. Los resultados sugieren que estos conocimientos pueden ser agrupados en ocho estándares: Fundamentos, características de estudiantes con talento y su contexto, nociones curriculares, sistema de evaluación, estrategias instruccionales, proceso de identificación, rol profesional y trabajo colaborativo. Se discuten las implicancias, a nivel nacional y latinoamericano, de contar con estándares de formación en este campo de la educación.
\end{abstract}

Palabras clave: estándares, saberes, formación de profesores, educación de talentos.

\begin{abstract}
This paper presents a qualitative investigation with the purpose of generating a proposal for standards in the teacher training process for the education of academically talented children. Four phases were conducted with the goal of obtaining the 'know' and 'know how to do' in the education of professionals in talented education. The results suggest that these knowledge's can be grouped in eight standards: Foundations, characteristics of gifted students and their context, notions of curriculum, assessment system, instructional strategies, identification process, professional role and collaborative work. We hold the discussion, at a national and Latin-American level, of the implications of having training standards in this particular field of education.
\end{abstract}

Key Words: standards, knowledges, teacher training, talents' education.

\section{Resumo}

Apresentam-se os resultados de uma pesquisa qualitativa, cujo objetivo foi gerar uma proposta de critérios centrais para formação de professores da educação de estudantes com aptidão para a aprendizagem. Quatro fases realizadas permitiram obter os "saberes" e o "saber como" a serem considerados na formação profissional docente. Os resultados sugerem que esses conhecimentos sejam agrupados de acordo com oito critérios: fundamentos, características de estudantes com aptidão para aprendizagem e seu contexto, noções curriculares, sistema de

Este artículo se deriva de la investigación postdoctoral de la autora, referida a los saberes y saberes hacer que debe poseer un profesor o profesional de la educación que trabaje con niños y niñas con talento académico. Dicha investigación se realizó en el Centro de Estudios y Desarrollo de Talentos de la Pontificia Universidad Católica de Chile - Proyecto de Inserción PSD71: Fortalecimiento del área de Investigación del Centro de Desarrollo de Talentos-PENTA UC: área cognitiva, socioafectiva y educativa. 
avaliação, estratégias de ensino, processo de identificação, papel profissional do professor e trabalho colaborativo. São discutidas, no âmbito nacional e latino-americano, as implicações da existência de critérios para a formação docente nessa área da educação.

Palavras chave: padrões, saberes e saber como, formação de professores, educação de estudantes com aptidão para aprendizagem.

\section{INTRODUCCIÓN}

En la última década, las reformas educativas gestadas e implementadas tanto a nivel internacional como latinoamericano se han centrado en identificar y caracterizar lo que deben saber y saber hacer los estudiantes al finalizar una etapa escolar. Para ello, dichas reformas han planteado dos tipos de estándares a saber: (a) de contenido, cuyo propósito ha sido clarificar aquellos conocimientos, habilidades y disposiciones que los estudiantes deben conocer y ser capaces de poner en uso al finalizar un ciclo escolar, y (b) de desempeño, que permiten observar, describir y evaluar los niveles de progresión de los estudiantes en el alcance de sus aprendizajes.

En algunos países latinoamericanos, como Colombia y Chile, dichos estándares han alimentado los procesos de formación inicial y continua de los profesores (Cox; 2008; Ferrer, 2007; MINEDUC, 2003). Particularmente en Chile, desde el año 2000, la evaluación docente es un tema que comienza a preocupar la agenda pública, así como la creación de estándares de formación para profesores (Redondo et al. 2004). A la fecha se cuenta con estándares de formación de profesores a nivel inicial (MINEDUC, 2000), estándares que se focalizan en los procesos de enseñanza-aprendizaje a los que se ve enfrentado un profesor en ejercicio de su tarea de enseñar (MINEDUC, 2003), y recientemente se está llevando a cabo un proceso de construcción de estándares disciplinarios (CEPPE, 2010). Ahora bien, si partimos del hecho de que a nuestras aulas asiste una gran diversidad de alumnos y teniendo en cuenta que sustentamos que el ejercicio de plantear estándares de contenido y desempeño permitiría a los docentes atender las individualidades y particularidades de los estudiantes, se hace evidente la necesidad de formar profesores en procesos pedagógicos que les permitan entender y atender dicha diversidad. Pensar en atender la diversidad implicaría por tanto, además de las iniciativas ya mencionadas, generar estándares de formación profesional en los que se considere población con necesidades educativas especiales tanto como con potenciales intelectuales altos.

Una revisión de la literatura latinoamericana a la cual se tuvo acceso nos permitió evidenciar que en muchos países la educación de talentos es un tema relativamente nuevo y los estudios encontrados se centran en temáticas como procesos de identificación, implementación de programas para niños con talento, pero no directamente sobre los saberes que debiese poseer un profesional en este campo (Benavides et al. 2004). En Chile y específicamente el Centro de Estudios y Desarrollo de Talentos, ha venido trabajando desde el 2001 en la generación de programas de enriquecimiento intra y extraescolares para estudiantes con talento académico, así como en modalidades de formación presencial y a distancia para profesores y profesionales interesados en este campo. Particularmente en esta última modalidad, se evidencia el interés de profesionales latinoamericanos que reportan la carencia de programas de formación en sus países que les brinde los saberes necesarios para trabajar con estudiantes talentosos. Asimismo, dicha revisión nos acercó solo a algunas descripciones en las que se explicitan características y competencias que 
debiese poseer un profesor en educación de talentos (Casillas, 1998; Rodríguez, 2006), no así investigaciones que planteen estándares de formación docente.

Dar respuesta a preguntas como ¿Qué saberes debe poseer un profesor para reconocer al estudiante talentoso dentro de la diversidad? ¿Cómo puede saber un profesor que uno de sus estudiantes está en un nivel sobresaliente, por encima de su propio grupo de pares? ¿Cómo puede continuar potenciando el aprendizaje de este estudiante?, requiere ciertamente de un conjunto de saberes que, planteados en una estructura de estándares, favorezcan la formación de profesores que asuman el desafío de enseñar a niños niñas y jóvenes con talento.

En este artículo se presenta una investigación cuyo objetivo fue generar una propuesta de estándares iniciales que aseguren procesos de formación de profesores en este campo de la educación, sustentados en las modalidades de perfeccionamiento que desarrollamos en el Área de Educación y Capacitación del Centro de Estudios y Desarrollo de Talentos-PENTA UC. Asimismo, pretendemos que éstos favorezcan la construcción de un diálogo compartido a nivel latinoamericano en relación a aquellos saberes necesarios de considerar si se quiere contar con profesores que potencien el talento académico en aquellos niños que lo poseen. A continuación se indica brevemente qué entendemos por talento académico así como los antecedentes generales que desde la literatura anglosajona nos permite dar sustento a nuestra propuesta de estándares.

\section{1. ¿QUÉ ENTENDEMOS POR TALENTO ACADÉMICO?}

Talento académico es un tipo específico de talento tal como existe el talento musical, artístico o deportivo. Para que una persona sea considerada académicamente talentosa debe poseer una habilidad o capacidad superior en las áreas académicas como la matemática, las ciencias naturales, sociales y/o en las humanidades. Es a este tipo de talento al que se han dirigido todos los programas de enriquecimiento intra y extraescolares implementados por el Centro de Estudios y Desarrollo de Talentos. Para nosotros, el talento académico puede ser general o específico. General, cuando las capacidades superiores se manifiestan en varias o todas las áreas académicas, y específico, cuando se presenta en una de ellas (Arancibia, 2009).

Consideramos que el talento académico se distribuye homogéneamente dentro de la población sin importar diferencias económicas, de género o raza, y reconocemos la necesidad de generar ambientes enriquecidos en los que se desafíe esta capacidad superior. No basta solamente con poseer esta capacidad para ser talentoso académicamente, por el contrario se debe contar con contextos adecuados de aprendizaje que potencien verdaderamente el talento, de lo contrario puede perderse. Maximizar el potencial de estudiantes con altas capacidades o talentosos al interior del aula regular como en programas especiales, requiere de profesionales formados en educación de talentos que reconozcan e implementen en la práctica aquellos saberes y saberes hacer necesarios para generar ambientes de aprendizaje desafiantes para sus estudiantes (Dixon y Moon, 2006).

\subsection{UNA REVISIÓN DE PROPUESTAS INTERNACIONALES}

Una vasta literatura anglosajona pone en evidencia aquellas características y competencias propias de profesores de niños talentosos (Bishop, 1968; Heath, 1997; Milgram, 
1979) las que, en muchas ocasiones se transforman en "un listado de virtudes que pueden caracterizar a todos los profesores o a todos los líderes", tal como lo afirma Feldhusen (1997, p. 548). Un trabajo reflexivo de diferenciación en este campo derivó, en los países anglosajones y más recientemente Europa y en especial los países germano-parlantes, al planteamiento de estándares de formación de profesores que diera soporte a una educación de calidad para niños con talento (Rosner, 2009; VanTassel-Baska y Johnsen, 2007).

Standards for Graduate Programs in Gifted Children, es una propuesta generada como orientación para la construcción de programas de alta calidad por National Association for Gifted Children (NAGC, 1995). Ésta indica los conocimientos tanto como las habilidades a potenciar en un programa de formación de profesionales en este campo. Aunque los 13 conocimientos y 17 habilidades que conforman esta propuesta no están agrupados por dimensiones, éstos responden a temáticas como fundamentos, características de los niños con talento, metodologías, sistemas de evaluación e identificación. Por ejemplo se destacan indicadores como el reconocimiento de las necesidades de los estudiantes y a partir de éste, variar las estrategias de enseñanza que permitan responder a las necesidades individuales.

En el 2007 VanTassel-Baska et al., publican el artículo Teacher Education Standards for the Field of Gifted Education, una propuesta integradora para la formación de profesionales en el siglo XXI. Esta propuesta contiene 70 indicadores agrupados en 10 estándares de contenido, a saber, los fundamentos, características de los aprendices, diferencias de aprendizaje individual, estrategias instruccionales, ambientes de aprendizaje e interacciones sociales, lenguaje y comunicación, planificación instruccional, evaluación, práctica ética y profesional y colaboración (p. 184). Esta propuesta de estándares comparte un vasto conjunto de investigaciones, literatura y estudios prácticos que evidencian mejores prácticas en la educación de dotados con un fuerte énfasis en la diversidad. Asimismo, nos muestra cómo a través del consenso entre organizaciones que lideran la formación de profesionales en este campo, universidades, profesionales expertos así como profesores, se puede llegar a un planteamiento de estándares que definen conocimientos y habilidades esenciales para la formación inicial de profesores en la educación de talentos.

Recientemente, otra propuesta sobre estándares en este campo de la educación es planteada desde los países germano-parlantes que desde la agrupación International Panel of Expert for Gifted Education, establecen 6 áreas de competencias que caracterizan los estándares de cualificación para profesores. Conocimiento básico sobre el statu quo científico en educación de talentos, trabajo científico-empírico, diagnóstico e identificación, enseñanza y aprendizaje de los dotados y talentosos, fundamentos para el asesoramiento y apoyo, desarrollo instruccional e institucional, han sido formulados luego de clarificar aquellas funciones que el profesor tiene dentro y fuera de la escuela regular (Rosner, 2009).

Las propuestas señaladas anteriormente son un aporte para nuestro estudio, primero porque confirman la necesidad de contar con estándares de formación contextualizados, que certifiquen la capacidad de los profesores de afrontar las exigencias y desafíos de educar niños con talento. Segundo, el reconocer los estándares como un conjunto de conocimientos y habilidades que se deben evidenciar en la práctica, y tercero, la relevancia de considerar estándares de formación que potencien las funciones del profesor al interior del aula como dentro de la escuela. 


\section{3. ¿QUÉ DEBE SABER Y SABER HACER UN PROFESOR QUE ENSEÑE A NIÑOS CON TALENTOS?}

Un amplio cuerpo teórico y empírico fundamenta esta pregunta sobre los saberes y saberes hacer necesarios de poseer si se quiere enseñar a niños con talento. Reconocer las características cognitivas y socioemocionales de los niños con talento así como los fundamentos conceptuales y empíricos que sustentan dichas diferencias, es un indicador clave en el conjunto de saberes que debe poseer un profesor (Dixon et al., 2006; Preckel, et al. 2008). Los estudiantes con talento académico establecen relaciones conceptuales con diferentes campos del conocimiento, guían sus procesos de aprendizaje al trabajar en una tarea (PENTA UC, 2000) y pueden presentar alta intensidad y sensibilidad emocional (Sánchez y Flores, 2006). Talento y dificultades de aprendizaje no son excluyentes, pueden coexistir, por lo que el profesor debe saber reconocer esta condición tal como poder generar estrategias que permitan relevar el talento y apoyar la dificultad (Dixon et al., 2006; Assoline, et al. 2009). Los contextos familiar, escolar y social juegan un rol preponderante en el desarrollo del talento (Campell y Verna, 2007) tal como lo avala la literatura reportada por VanTassel-Baska et al. (2007). Por ello, el profesor debe conocer y reconocer las diferentes dinámicas que pueden darse en estos contextos con el fin de intervenir apropiadamente en pro del desarrollo del talento (PENTA UC, 2008; Sánchez et al. 2006).

Ciertamente los profesores deben contar con un conjunto de conocimientos sólidos en torno a las concepciones, teorías y fundamentos del estudio del talento que les permita dar sustento a: su conceptualización del talento (Colangelo y Davis, 1997; Gagné, 2003; Mönks y Monson, 2000; Renzulli, 1978), cómo implementar procesos de identificación de estudiantes con talento, así como el plantear programas de excelencia (Colangelo et al., 1997; Yoon y Gentry, 2009). Asimismo, los profesores deberían contar con conocimientos generales acerca de la educación de talentos en el mundo, así como las políticas públicas que se han generado a nivel latinoamericano y/o internacionalmente, que apuntan a brindar educación de calidad a este grupo de estudiantes (Mönks y Pflüger, 2005; Ministerio Educación de Colombia, 2006).

El profesor de niños con talento requiere un conjunto especial de conocimientos y habilidades que potencien en ellos sus habilidades, a través de contenidos complejos y desafiantes (Croft, 2003). Para ello, el profesor debe construir espacios de aprendizaje enriquecedores en los que genere un clima positivo de enseñanza y aprendizaje y le permita al estudiante ser autónomo en su trabajo (Hansen y Feldhusen, 1994). Construir este tipo de ambientes enriquecidos, requiere conocimiento de fundamentos, orientaciones y principios curriculares apropiados para la educación de talentos, modelos pedagógicos (Maker y Nielson, 1995; VanTassel-Baska, 2000), estrategias instruccionales que enriquezcan el talento en los estudiantes y continúen potenciando en ellos sus habilidades (Baldwin y Coleman, 2000; Betts, 2004; Cabrera, 2010; Dixon et al., 2006; Graffam, 2001; Joffe, 2001; Miranda y Landmann, 2001), tanto como el uso de variadas formas de evaluación que permitan recoger los aprendizajes alcanzados, retroalimentar al estudiante en su proceso y realizar ajustes en la práctica de acuerdo a sus particularidades. Para ello se recomienda el uso de múltiples fuentes formativas y sumativas tales como rúbricas, portafolios, o modelos basados en la ejecución (Siegle, 2000; Tomlinson, 1999; Sternberg y Grigorenko, 2000; VanTassel-Baska, 2002).

Nuestro profesor debe tener conocimientos base del proceso de identificación y selección que le permitan entender dicho proceso y poder participar en la aplicación de 
metodologías cualitativas (Colangelo et al., 1997; Sternberg y Subotnik, 2000). Dicho conocimiento también le permitirá comunicarse efectivamente con otros profesionales que estén a cargo del proceso de identificación y selección. En este entendido, el profesor debe contar con las competencias necesarias para comunicarse efectivamente con otros actores (padres, profesionales) así como con las habilidades interpersonales necesarias para trabajar colaborativamente (Campell et al, 2007). Finalmente, el profesor debe conocer aquellas características y competencias que se espera despliegue al educar niños con talento. Un conocimiento de este tipo le permitirá, en conjunto con los otros saberes y saberes hacer ya enunciados, evaluar e identificar diferencias en su función como profesor de estudiantes con talento con respecto a un profesor regular (Abel y Karnes, 1994; Chan, 2001; Croft, 2003; McGinty, 2010; Mettrau, 2010; Vialle y Quigley, 2002).

Educar a niños con talento requiere formar profesores que cuenten con los conocimientos y habilidades que les permita brindar a los estudiantes con talento una educación de calidad acorde a sus necesidades. Bajo esta premisa y considerando la necesidad de estándares de formación a nivel nacional y latinoamericano, el objetivo central de esta investigación fue generar una propuesta de estándares iniciales que aseguren procesos de formación de profesores en el campo de la educación de talentos académicos. Esta propuesta, aunque comparte algunos estándares planteados a nivel internacional, presenta una configuración propia y contextual a nuestra realidad sustentada en la literatura y en las modalidades de formación implementadas en el Centro, así como el consenso entre expertos y profesionales formados en el área. Se describe el diseño de la investigación así como los resultados, los que se discuten a la luz del marco teórico.

\section{MÉTODO}

En este estudio asumimos un enfoque metodológico cualitativo y usamos el método de análisis textual (Silverman y Marvasti, 2008). Esta investigación se realizó a partir de cuatro fases, las que se detallan a continuación:

\subsection{FASE 1}

Usando la técnica de análisis de contenido, se analizaron los programas de los tres diplomados de formación impartidos por el área de Educación y Capacitación del Centro de Estudios, así como el material que conforma cada uno de los 5 módulos que se imparten en la modalidad a distancia, vale decir, guías de contenido, de trabajo y material complementario que da fundamento a estos módulos. Estos materiales recogen las temáticas que también se abordan en las modalidades presenciales impartidas. El propósito fue extraer aquellos saberes y saberes hacer contenidos en este material, y que se han potenciado en los profesores capacitados a través de las diferentes modalidades.

Dos expertos en educación de talentos, quienes han participado ya sea en la construcción del material y/o impartiendo clases en estas modalidades de formación, analizaron los documentos y codificaron por separado estos saberes y saberes hacer en su correspondiente plantilla de vaciado de información. Finalizado este proceso, se integraron los dos análisis realizados, de tal manera de contar con un primer borrador que articulaba las coincidencias tanto como las no coincidencias en torno a ambos saberes, realizando así 
una primera reducción de la información. Posteriormente con éstos se realizó un ejercicio de agrupación, con el propósito de buscar lo que tenían en común, y así poder agruparlos bajo una categoría que pudiese caracterizar ese conjunto de saberes y saberes hacer.

\subsection{FASE 2}

Paralelo a la fase 1 se implementó la fase 2. Una encuesta, plasmada en formato on line, nos permitió indagar en la totalidad de profesionales capacitados durante los años 2009-2010 en la modalidad a distancia aquellos saberes y saberes hacer que ellos consideraron obtuvieron luego del proceso de formación. Se contó con la información de 33 profesionales, de los cuales 22 eran profesores, 8 psicólogos y 3 de otras profesiones. Dentro de este grupo se capacitaron dos profesoras, de Argentina y Costa Rica. Al igual que en la fase 1, la información fue analizada usando el análisis de contenido, esta vez, por uno de los expertos que tuvo la tarea de cruzar el conjunto de saberes explicitado por los profesionales con los obtenidos inicialmente. En esta fase se obtuvo un primer borrador de estándares, agrupados bajo ciertas categorías que permitían caracterizar los saberes obtenidos hasta el momento. Cabe señalar que durante la fase 1 y 2 a la par los expertos contrastaban los resultados obtenidos con la literatura conocida sobre el campo de educación de talentos.

\subsection{FASE 3}

Los saberes y saberes hacer categorizados en las fases anteriores fueron ordenados en un instrumento tipo encuesta. Ésta tenía tres propósitos: (a) recoger la evaluación de cuan necesario o innecesario era considerar cada uno de estos saberes cuando se habla de contar con profesores que se desempeñan efectivamente en educación de talentos, (b) indicar qué categoría asignarían a cada grupo de los estándares presentados y, (c) evaluar si alguno de los saberes o saberes hacer estaba contenido en alguno de los ya planteados. Esto, con la finalidad de validar las categorías iniciales reportadas en la fase 2. Dicho instrumento fue completado por 10 profesionales, 8 psicólogos, 1 teólogo y 1 ilustradora, quienes en promedio han trabajado un año y 8 meses en el tema de educación de talentos y forman parte del equipo de profesionales del Programa Educacional para niños con Talentos Académicos-PENTA UC del Centro de Estudio y Desarrollo de Talentos.

\subsection{FASE 4}

La evaluación dada por los profesionales fue usada como insumo para la ejecución de la última fase. Los dos expertos de la fase 1 trabajaron esta vez en verificar cuán fiable fue la propuesta de los saberes y saberes hacer, así como la categorización recibida a partir de las evaluaciones del equipo del programa. Las 8 dimensiones establecidas como grupos de estándares fueron validadas por todos los profesionales y se alcanzó en promedio un $98 \%$ de acuerdo. Un ejercicio de ordenamiento y depuración al interior de los saberes y saberes hacer de cada estándar fue realizado por parte de los expertos en forma conjunta, a partir de la evaluación de los profesionales del equipo PENTA, cuando los saberes habían sido indicados por ellos como contenidos en otros o bien, si se consideraba innecesario en la formación de profesores por su no pertinencia con su 
función. Finalmente se obtuvieron los estándares de formación de profesores en educación de talentos que se detallan en la siguiente sección.

\section{RESULTADOS}

Los resultados que se presentan a continuación evidencian aquellos conocimientos de base que el el profesor debe poseer (saberes) y aquellos saberes puestos en uso que deben evidenciarse en su práctica pedagógica (saber hacer) y que son necesarios de considerar en la formación de profesores y profesionales interesados en la educación de talentos. Estos se encuentran agrupados en ocho dimensiones que conforman los diferentes estándares de formación a saber: Fundamentos, Características del Estudiante con Talento y sus Contextos Cercanos, Nociones Curriculares, Sistema de Evaluación, Estrategias Instruccionales, Proceso de Identificación y Selección, Rol Profesional y Trabajo Colaborativo.

\subsection{FUNDAMENTOS}

El profesor debe manejar conocimientos de base desde una perspectiva histórica, política, sociocutural psicológica y educativa que lo ubiquen en el campo de la educación de talentos. Esto le permitirá tener fundamentos conceptuales claros y bases sólidas en relación a teorías, modelos, políticas que dan sustento a la educación de talentos, así como una postura crítica frente al campo en el que se desempeñará.

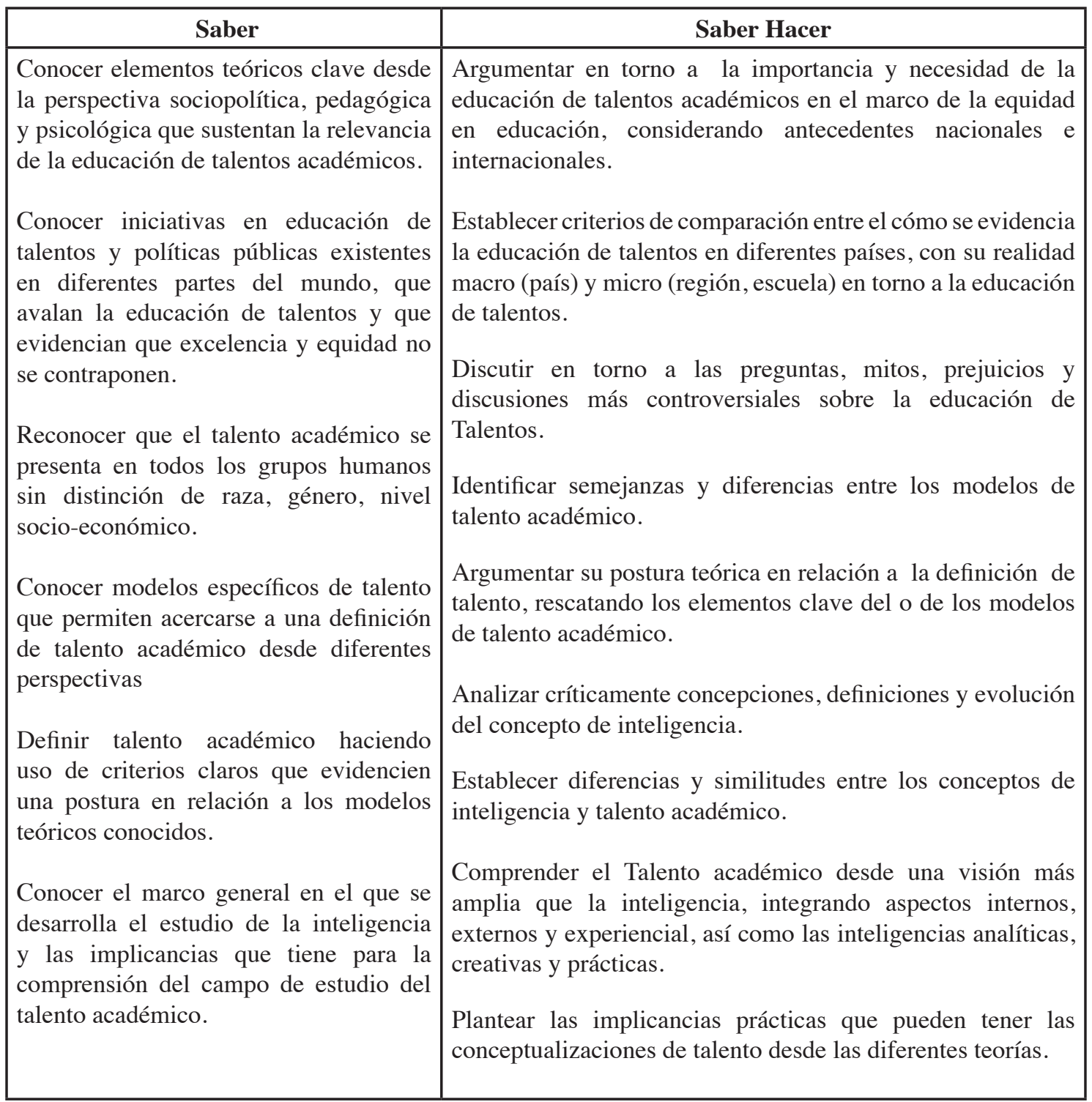




\subsection{CARACTERÍSTICAS DEL ESTUdiANTE CON TALENTO y SUS CONTEXTOS CERCANOS}

Conocer las características de los estudiantes con los que se trabaja es condición necesaria para enseñar a niños con talento. Reconocer las características particulares del estudiante talentoso en los planos cognitivo, afectivo y social, en las diferentes edades (preescolar-escolar: básica y media), posibles dificultades individuales que pueden entorpecer su proceso de aprendizaje, así como las características de su contexto familiar, escolar y social posibilita una intervención educativa más efectiva para el profesor que trabajará en este campo. Se hace necesario que el profesional capacitado pueda evidenciar las relaciones que se establecen es estos diferentes dominios para brindar una práctica efectiva a los estudiantes con talento.

\begin{tabular}{|c|c|}
\hline Saber & Saber Hacer \\
\hline $\begin{array}{l}\text { Reconocer la heterogeneidad al interior del grupo de } \\
\text { niños con talento en relación a su talento. } \\
\text { Reconocer que este grupo de niños(as) y jóvenes } \\
\text { con talento académico requieren de estrategias de } \\
\text { apoyo adicionales de acuerdo a sus necesidades } \\
\text { particulares. } \\
\text { Conocer las características de los alumnos con } \\
\text { talentos académicos en el plano de las relaciones } \\
\text { sociales que establecen con sus pares, docentes y } \\
\text { miembros de la comunidad. } \\
\text { Conocer posibles características que pueden } \\
\text { evidenciarse en las relaciones sociales que establecen } \\
\text { los alumnos con talentos académicos. } \\
\text { Conocer y manejar conceptos relacionados con } \\
\text { la doble excepcionalidad y bajo rendimiento en } \\
\text { estudiantes con talentos académicos, especialmente } \\
\text { aquello que refiere a su definición, características, } \\
\text { implicancias y estrategias de apoyo. } \\
\text { Conocer dinámicas familiares y escolares (positivas } \\
\text { y/o negativas) que pueden darse al interior de estos } \\
\text { contextos, en los que un integrante es un niño con } \\
\text { talento académico. }\end{array}$ & $\begin{array}{l}\text { Describir un niño con talento académico en los } \\
\text { planos cognitivo, afectivo y social, evidenciando } \\
\text { posibles dificultades de aprendizaje, tanto como } \\
\text { sus relaciones familiares y sociales. } \\
\text { Identificar dinámicas y/o situaciones que viven } \\
\text { estudiantes con talentos académicos y que dicen } \\
\text { relación con sus características cognitivas y } \\
\text { socioafectivas. } \\
\text { Fomentar ambientes psicosociales favorables } \\
\text { para el desarrollo del talento académico en los } \\
\text { estudiantes que lo poseen. } \\
\text { Evaluar qué aspectos del contexto familiar, } \\
\text { escolar, social, posibilitan o entorpecen el } \\
\text { desarrollo del potencial en los alumnos talentosos. } \\
\text { Proponer posibles estrategias de apoyo y } \\
\text { orientación para niños (as) y jóvenes con talento } \\
\text { académico que pueden presentar o no alguna } \\
\text { dificultad educativa en beneficio de su desarrollo } \\
\text { cognitivo, afectivo y social,involucrando contexto } \\
\text { familiar, escolar y/o social. }\end{array}$ \\
\hline
\end{tabular}

\subsection{NOCIONES CURRICULARES}

En educación de talentos los principios, fundamentos y orientaciones curriculares que la sustentan, se diferencian de los propuestos en educación general. Por ello, este grupo de estándares da fundamento conceptual a la función de enseñar del profesor. Para que pueda desempeñarse efectivamente en el contexto educativo de niños con talento, un profesor debe considerar los saberes y saberes hacer que se detallan a continuación: 


\begin{tabular}{|c|c|}
\hline Saber & Saber Hacer \\
\hline $\begin{array}{l}\text { Conocer los modelos curriculares, así como } \\
\text { la organización y funcionamiento de tipos de } \\
\text { programas que comúnmente se enuncian en } \\
\text { educación de talentos. } \\
\text { Conocer los principios, fundamentos curriculares } \\
\text { y orientaciones filosóficas que sustentan el diseño } \\
\text { curricular en educación de talentos. } \\
\text { Conocer los componentes y etapas a considerar en } \\
\text { la planificación y desarrollo de un currículum para } \\
\text { estudiantes con talentos académicos. } \\
\text { Conocer los aspectos relevantes a considerar ante } \\
\text { el planteamiento de un programa o proyecto de } \\
\text { educación de talentos. }\end{array}$ & $\begin{array}{l}\text { Diferenciar los modelos curriculares que privilegian el } \\
\text { aprendizaje de contenidos, el desarrollo de habilidades o } \\
\text { aquellos que centran sus experiencias educativas en base } \\
\text { a metaconceptos o temáticas transdisciplinarias. } \\
\text { Reconocer las modalidades curriculares y/o tipo de } \\
\text { programa que se encuentran a la base de diferentes } \\
\text { propuestas de educación de talentos académicos a nivel } \\
\text { nacional e internacional. } \\
\text { Explicitar similitudes y diferencias entre una clase } \\
\text { regular y una clase para estudiantes con talentos } \\
\text { considerando los componentes curriculares (contenidos, } \\
\text { procesos y productos, metas y objetivos, temas, } \\
\text { problemas y conceptos, actividades y materiales. } \\
\text { Juzgar la calidad y pertinencia de un currículum para } \\
\text { estudiantes con Talentos académicos. } \\
\text { Relacionar las características y necesidades educativas de } \\
\text { estudiantes con talentos Académicos con la formulación } \\
\text { de metas de aprendizajes. } \\
\text { Reconocer el impacto de los programas en el estudiante } \\
\text { talentoso, la familia, los profesores así como al contexto } \\
\text { educativo general. } \\
\text { necesidades y características de niños (as) y/o jóvenes } \\
\text { con talentos académicos adecuadas así como al contexto. }\end{array}$ \\
\hline
\end{tabular}

\subsection{SISTEMA DE EVALUACIÓN}

Los profesores en educación de talentos deben reconocer la evaluación como un proceso dinámico, continuo e integrado desde la etapa de planificación, implementación y finalización del proceso educativo. Incorporar la evaluación como un elemento central en todas las etapas permite: (a) el ajuste de la unidad de enseñanza o curso a implementar, (b) evaluar las estrategias instruccionales que hacen parte de su práctica pedagógica y que permiten potenciar el desafío cognitivo en sus estudiantes y, (c) el reconocimiento del progreso y avance de los estudiantes en su proceso de aprendizaje.

\begin{tabular}{|l|l|}
\hline \multicolumn{1}{|c|}{ Saber } & \multicolumn{1}{|c|}{ Saber Hacer } \\
\hline $\begin{array}{l}\text { Conocer las características, criterios, funciones y } \\
\text { relevancia de la evaluación en el proceso de enseñanza- } \\
\text { aprendizaje de los estudiantes con talentos académicos. }\end{array}$ & $\begin{array}{l}\text { Proponer cursos o unidades de enseñanza que } \\
\text { consideren las condiciones y componentes } \\
\text { curriculares requeridos en educación de talentos. }\end{array}$ \\
$\begin{array}{l}\text { Conocer indicadores qué permiten evaluar si una unidad } \\
\text { de enseñanza es adecuada o no para ser aplicada en } \\
\text { educación de talentos. }\end{array}$ & $\begin{array}{l}\text { Evaluar si una unidad de enseñanza es adecuada } \\
\text { para ser implementada con estudiantes con talento } \\
\text { académico a partir de criterios establecidos (p.e. } \\
\text { reconocimiento de habilidades, existencia de } \\
\text { aprendizaje autodirigidos, etc.). }\end{array}$ \\
$\begin{array}{l}\text { Reconocer los objetivos de aprendizaje, momentos de } \\
\text { evaluación, instrumentos, métodos de evaluación y } \\
\text { retroalimentación como los principales elementos de } \\
\text { considerar al plantear un sistema de evaluación. }\end{array}$ & \\
\hline
\end{tabular}




\begin{tabular}{|c|c|c|}
\hline & & $\begin{array}{l}\text { Diseñar o seleccionar sistemas de evaluación } \\
\text { coherentes con los objetivos de aprendizaje, que } \\
\text { considere las necesidades particulares de los niños } \\
\text { con talento y que permita registrar los progresos de } \\
\text { sus estudiantes. } \\
\text { Plantear los momentos en los cuales evaluará los } \\
\text { aprendizajes de sus estudiantes talentosos. } \\
\text { Evaluar para quienes y para qué es útil la información } \\
\text { que recoja en cada uno de los momentos del proceso } \\
\text { de evaluación. }\end{array}$ \\
\hline
\end{tabular}

\subsection{ESTRATEGIAS INSTRUCCIONALES}

Para que el proceso de enseñanza evidencie resultados positivos en los aprendizajes de los estudiantes, el profesor debe conocer y hacer uso de estrategias que le permitan generar un ambiente de aprendizaje acorde a las características de estudiantes con talento. Por ello, el profesor debe estar en capacidad de implementar estrategias en las que el desafío cognitivo responda a las características cognitivas de sus estudiantes y que, permita potenciar el desarrollo de habilidades de pensamiento crítico, creativo tanto como sus habilidades socio-afectivas.

\begin{tabular}{|c|c|}
\hline Saber & Saber Hacer \\
\hline $\begin{array}{l}\text { Conocer diferentes modelos o propuestas } \\
\text { pedagógicas en torno al desarrollo de habilidades } \\
\text { superiores y cómo pueden usarse en la construcción } \\
\text { de procesos de enseñanza desafiantes para los niños } \\
\text { con talentos académicos. } \\
\text { Conocer estrategias instruccionales y elementos clave } \\
\text { del clima de aprendizaje que apoyan y promueven en } \\
\text { los estudiantes su talento académico, y su desarrollo } \\
\text { socioemocional propiciando de esta manera un proceso } \\
\text { de enseñanza activo y diferenciado. } \\
\text { Reconocer la importancia de considerar el desafío, la } \\
\text { complejidad conceptual, altas expectativas, autonomía, } \\
\text { etc., como condiciones relevantes de relevar en la } \\
\text { educación de talentos académicos. } \\
\text { Conocer técnicas de cuestionamiento que potencien } \\
\text { el desarrollo de habilidades de orden superior, la } \\
\text { metacognición y la creatividad en los estudiantes con } \\
\text { talentos académicos. } \\
\text { Reconocer como el considerar las necesidades } \\
\text { particulares de los estudiantes talentosos, el uso de } \\
\text { condiciones y estrategias instruccionales apropiadas } \\
\text { potencian ambientes de aprendizaje que favorecen el } \\
\text { talento tanto como el conocimiento y la creatividad. }\end{array}$ & $\begin{array}{l}\text { Usar estrategias de enseñanza y metodologías } \\
\text { activas adecuadas para trabajar con estudiantes } \\
\text { con talentos académicos, que potencien el } \\
\text { desarrollo de sus habilidades. } \\
\text { Realizar adaptaciones curriculares que les permita } \\
\text { a los niños talentosos avanzar a su propio ritmo y } \\
\text { estilo de enseñanza. } \\
\text { Fortalecer las habilidades de orden superior, } \\
\text { procesos metacognitivos y habilidades socio- } \\
\text { afectivas en los estudiantes talentosos. } \\
\text { Generar instancias de aprendizaje en las que el } \\
\text { desafío cognitivo es su eje articulador. } \\
\text { Plantear estrategias instruccionales que potencien } \\
\text { el desarrollo cognitivo de los niños con talento } \\
\text { académico que presentan dificultades de } \\
\text { aprendizaje. }\end{array}$ \\
\hline
\end{tabular}




\subsection{PROCESO DE IDENTIFICACIÓN Y SELECCIÓN}

El profesor en educación de talentos debe tener una base de conocimientos sólidos en torno a los procesos de identificación y selección. Esto con el propósito de que pueda interactuar con el grupo de profesionales encargado de realizar el proceso de identificación y selección, así como tomar decisiones al interior de su comunidad escolar que faciliten la nominación de estudiantes con talento.

\begin{tabular}{|c|c|}
\hline Saber & Saber Hacer \\
\hline $\begin{array}{l}\text { Reconocer la definición de talento como el } \\
\text { punto de partida y eje articular del proceso de } \\
\text { identificación y selección. } \\
\text { Conocer los supuestos, principios y pasos } \\
\text { a considerar del proceso de identificación y } \\
\text { selección, así como las implicancias que tiene } \\
\text { el llevar a cabo en forma adecuada o no dicho } \\
\text { proceso. } \\
\text { Conocer, de manera general, las principales } \\
\text { metodologías, cualitativas y cuantitativas, usadas } \\
\text { para el proceso de identificación y selección de } \\
\text { estudiantes con talentos académicos. } \\
\text { Reconocer la baja representación de los sectores } \\
\text { más desaventajados como un problema que } \\
\text { puede presentarse en el proceso de identificación } \\
\text { y selección. } \\
\text { Conocer los distintos criterios o aproximaciones } \\
\text { comúnmente usados en el proceso de } \\
\text { identificación y selección de estudiantes con } \\
\text { talentos académicos, así como sus ventajas y } \\
\text { desventajas. } \\
\text { Reconocer la necesidad de personal capacitado } \\
\text { para llevar a cabo el proceso de selección e } \\
\text { identificación de niños con talento. }\end{array}$ & $\begin{array}{l}\text { Usar metodologías cualitativas que enriquezcan el } \\
\text { proceso de selección e identificación de niños con } \\
\text { talento académico. } \\
\text { Generar una postura personal crítica, informada } \\
\text { y argumentada respecto al problema de la baja } \\
\text { representación de sectores sociales, económicos y } \\
\text { culturalmente desventajados en la identificación y } \\
\text { selección de estudiantes con talentos académicos. }\end{array}$ \\
\hline
\end{tabular}




\subsection{ROL PROFESIONAL}

Un profesor de educación de talentos, al igual que el de educación general, debe reconocer la relevancia de su rol como profesor en el proceso de enseñanza-aprendizaje de estudiantes con talentos académicos. Por ello, se hace necesario que reconozca aquellas características y competencias que lo harán desempeñarse efectivamente en este campo educativo.

\begin{tabular}{|l|l|}
\hline Saber & Saber Hacer \\
\hline $\begin{array}{l}\text { Conocer rasgos distintivos (características y } \\
\text { competencias) que caracterizan el desempeño } \\
\text { exitoso de un profesor de niños (as) y jóvenes } \\
\text { con talentos académicos y que favorecen el } \\
\text { aprendizaje de estudiantes con talento. }\end{array}$ & $\begin{array}{l}\text { Evaluar su propia concepción de educación } \\
\text { de talentos académicos y cómo ésta facilita o } \\
\text { entorpece su práctica pedagógica. }\end{array}$ \\
\hline $\begin{array}{l}\text { Conocer antecedentes generales, empíricos y/o } \\
\text { teóricos, sobre el sistema educacional regular, } \\
\text { especialmente en lo que respecta a la cultura } \\
\text { docente. }\end{array}$ & $\begin{array}{l}\text { Reflexionar en torno a aquellas acciones que } \\
\text { favorecen y garantizan experiencias educati- } \\
\text { académicos. }\end{array}$ \\
\hline & $\begin{array}{l}\text { Establecer vínculos afectivos sólidos con sus } \\
\text { estudiantes talentosos. }\end{array}$ \\
\hline
\end{tabular}

\subsection{TRABAJO COLABORATIVO}

El profesor formado en educación de talentos debe estar en capacidad de trabajar colaborativamente en su comunidad escolar con otros profesionales tanto como con padres. Sus competencias comunicativas e interpersonales se ponen en juego en la práctica, con el propósito de generar ambientes de aprendizaje apropiados a sus estudiantes.

\begin{tabular}{|l|l|}
\hline Saber & Saber hacer \\
\hline $\begin{array}{l}\text { Reconocer la importancia de trabajar en } \\
\text { equipo (profesores y diseñadores de currículo) } \\
\text { en la construcción e implementación de una } \\
\text { unidad de enseñanza aprendizaje en educación } \\
\text { de talentos académicos. }\end{array}$ & $\begin{array}{l}\text { Trabajar en equipo con otros profesionales, } \\
\text { aportando desde su rol de profesor en los dife- } \\
\text { rentes procesos en los que participe. }\end{array}$ \\
\hline & $\begin{array}{l}\text { Establecer adecuadas relaciones interpersonales } \\
\text { y canales de comunicación con otros actores en } \\
\text { el proceso (padres, profesores, alumnos, etc.). }\end{array}$ \\
\hline & $\begin{array}{l}\text { Comunicar estrategias de apoyo a colegas, de } \\
\text { manera de privilegiar el trabajo con estudian- } \\
\text { tes con talento académico. }\end{array}$ \\
\hline & $\begin{array}{l}\text { Comunicar estrategias de apoyo a colegas, de } \\
\text { manera de privilegiar el trabajo con estudian- } \\
\text { tes con talento académico. }\end{array}$ \\
\hline
\end{tabular}




\section{DISCUSIÓN}

Esta investigación presenta los saberes y saberes hacer que se consideran necesarios en los procesos de formación de profesores interesados en el campo de la educación de talentos. Las 8 dimensiones en las que se estructuran los estándares obtenidos en nuestra investigación, son coincidentes con el planteamiento general de países como Estados Unidos, Inglaterra y los países germano-parlantes (Rosner, 2009; National Quality Standards in Gifted and Talented Education, 2005); VanTasel-Baska et al., 2007). Por ejemplo, algunos de los saberes y saberes hacer obtenidos desde los profesionales capacitados a distancia, y que fueron insumo en la fase 2, explicitan la necesidad de conocer las características de los estudiantes talentosos tanto como estrategias instruccionales o de evaluación que les permita potenciar el talento en sus estudiantes (Sánchez et al., 2006; VanTasel-Baska, 2000).

Creemos que los estándares que se presentan, producto de la investigación realizada, son una herramienta valiosa a nivel latinoamericano en la formación de profesionales desde programas generados en universidades o centros que trabajan en la educación de talentos, así como en la formación continua de profesores que quieren especializarse en este campo, o bien enriquecer sus prácticas pedagógicas en aula regular. Coincidimos con VanTasel-Baska et al. (2007) en que una propuesta de estándares permitiría unificar procesos de formación en este campo a nivel general en una región, así como convertirse en una herramienta que abra caminos en la construcción de políticas públicas que consideren la educación de talentos. Por tanto, creemos que contar con esta primera aproximación de estándares de formación de profesores en educación de talentos permitirá, a nivel macro, abrir puertas para establecer un diálogo común entre profesionales interesados en este campo, que favorezcan en los diferentes países latinoamericanos -incluido Chile- la generación de políticas públicas que trabajen en pro de la acreditación de profesionales en este campo.

Contar con estos estándares también permitirá a las universidades la generación de programas de formación continua a profesionales interesados en la educación de talentos tanto como la inclusión de algunos de estos estándares en la formación de profesores en los niveles inicial, básica y media, así como complemento a la formación de educadores diferenciales. Cualitativamente podemos indicar cómo profesionales de estos campos han reportado un cambio de paradigma no solo al reconocer que existen en sus aulas niños con talentos, sino también en su forma de enseñar al interior del aula regular.

De igual manera reconocemos que esta propuesta de estándares podría ser considerada como muy general y poco específica. Por ello, consideramos que esta primera propuesta de formación inicial puede y debe ser complementada con descripciones que acompañen los diferentes saberes y saberes hacer, que permitan caracterizar los desempeños que se pueden alcanzar en cada uno, así como con diferentes instrumentos o formas de evaluación que nos permitan evidenciar el alcance de dichos saberes.

Finalmente, consideramos que los estándares son un gran paso en el reconocimiento de estudiantes con talentos académicos, que al igual que todos requieren recibir una educación de calidad. Esto es, recibir una educación acorde a sus necesidades y particularidades. Asimismo, consideramos que para asumir esta tarea los profesores deben estar capacitados para poder enfrentar este desafío. Una educación de calidad para niños con talentos requiere de profesores de excelente calidad profesional. 


\section{REFERENCIAS BIBLIOGRÁFICAS}

Abel, T. y Karnes, F.A. (1994). Teacher's preferences among the lower socioeconomic rural and suburban advantaged gifted students. Roeper Review, 17(1), 52-57.

Arancibia, V. (2009). La educación de alumnos con talentos: una deuda y una oportunidad para Chile. Santiago de Chile: Pontificia Universidad Católica de Chile.

Assouline, S., Foley, M. y Doobbay, A. (2009). Profoundly Gifted Girls and Autism Spectrum Disorder: A Psychometric Case Study Comparison. Child Quarterly, 53(2) 89-105.

Baldwin, C. y Coleman, C. (2000). Achievement Goal Orientation: Instructional Practices and Teacher Perceptions of Gifted and/or Academically Talented Students. Paper presented at the Annual Meeting of the American Educational Research Association (New Orleans, LA, April 24-28).

Benavides, M., Maz, A., Castro, E. y Blanco, R. (Eds). (2004). La educación de niños con talento en Iberoamérica. Santiago (Chile): Orealc/Unesco. Retrieved from http: //unesdoc.unesco.org/ images/0013/001391/139179s.pdf

Betts, G. (2004). Fostering autonomous learners through levels of differentiation. Roeper Review, 26(4), 190-191. Doi: 10.1080/02783190409554269

Bishop, W. (1968). Successful teachers of the gifted. Exceptional Children, 34(5), 317-325.

Cabrera, E.P. (2010). Describing teaching practices in talented Children Classrooms: A case study. Manuscrito presentado para publicación.

Campbell, J. y Verna, M. (2007). Effective Parental Influence: Academic home climate linked to children's achievement. Educational Research and Evaluation, 13(6), 501-519. doi:10.1080/13803610701785949

Chan, D. (2001). Characteristics and competencies of teachers of gifted learners: The Hong Kong teacher perspective. Roeper Review, 23(4), 197-202.

Casillas, M.A. (1996). Capacitación a docentes en el desarrollo de potenciales: una estrategia para elevar la calidad de la Educación. Retrieved from http://educar.jalisco.gob.mx/06/6casilla.html

Centro de Estudios de Políticas y Prácticas en Educación-CEPPE (2010, noviembre 2010). [Web] Retrieved from http://www.ceppe.cl/estandares-de-desempeno-inicial-docentes-educacion-media.

Colangelo, N. y Davis, G. (1997). Handbook of Gifted Education. Second Edition. Estados Unidos: Allyn and Bacon.

Cox, C. (2008, diciembre). Profesores y Desafío país: Formación Inicial. Seminario El valor del Profesor, Fundación Arauco. Concepción: Santiago de Chile www.fundacionarauco.cl/_file/ file_3888_presentación-cristián-cox.pdf

Croft, L.J. (2003). Teachers of the gifted: Gifted teachers. In Colangelo, N. y Davis, G. (Hrsg.), Handbook of gifted education (S. 558-571). New York: Allyn and Bacon.

Dixon, F. y Moon S. (2006). The Handbook of Secondary Gifted Education. Texas: Prufrock Press Inc.

Feldhusen, J. F. (1997). Educating Teachers for work with talented youth. In Colangelo, N. y Davis, G.A. (eds.), Handbook of Gifted Education (pp. 547-552). Boston: Allyn and Bacon (2da edición).

Ferrer, G. (2006). Estudio Comparado Internacional sobre Procesos de Elaboración e Implementación de Estándares e Implementación de Estándares de Currículum en América Latina. PREAL Retrieved from http://200.79.124.184/prieb/images/stories/estudio_comparado_internacional.pdf

Gagné, F. (2003). Transforming Gifts into Talents: The DMGT as a Developmental Theory. In Colangelo, N. y Davis, G.A. (Eds.), Handbook of gifted education ( $3^{\text {rd }}$ ed.), pp. 60-74. Boston: Allyn and Bacon.

Graffam, B. (2006) A Case Study of Teachers of Gifted Learners: Moving From Prescribed Practice to Described Practitioners. The Gifted Child Quarterly, 50(2), 119-189.

Hansen, J.B. y Feldhusen, J.F. (1994). Comparison of trained and untrained teachers of gifted students. Gifted Child Quarterly, 38(3), 115-121.

Heath, W. (1997). What are the most effective characteristics of teachers of the gifted? (ERIC Document Reproduction Service No. ED411665). 
Joffe, W. (2001). Investigating the acquisition of pedagogical knowledge: Interviews with a beginning teacher of the gifted. Rooper Review, 23(1), 219-226.

Maker, J. y Nielson, A. (1995). Teaching Models in Education of the Gifted. Austin, Texas: Pro-ed.

Mettrau, M. (2010). Programas académicos para la formación de profesores de alumnos con superdotación y talento. REIFOP, 13,1. Retrieved from http://www.aufop.com

Milgram, R. (1979). Perception of Teacher Behavior in Gifted and Nongifted Children. Journal of Educational Psychology, 71(1), 125-128.

Ministerio Educación Nacional de Colombia (2006). Orientaciones para la atención educativa a estudiantes con capacidades o talentos excepcionales. Retrieved from http://www.areandina. edu.co/bienestar/documentos/LINEAMIENTOS_TALENTOS_EXCEPCIONALES.pdf

Mineduc (2003). Attracting, Developing and Retaining Effective Teachers. OECD Activity. Country. Background Report for Chile. Santiago, Chile: Autor.

(2003). Marco para la Buena Enseñanza. Santiago de Chile: Autor. Retrieved from www. acreditaciondocente.cl/usuarios/.../doc/200312031457060.mbe.pdf

(2000). Estándares de Desempeño para la Formación Inicial de Docentes. Santiago de Chile: Autor. Retrieved from www.mineduc.cl/biblio/documento/Estandares_de_desempeno.pdf

Miranda, E. y Landmann, R. (2001). Gifted teachers creating gifted Classroom: One exceptional teacher, one exceptional classroom. Rooper Review, 23 (4), 230-234.

Mcginty, P. (2010). The influence of teacher characteristics on preference for models of teaching. (Doctoral Dissertation, Faculty of the Usc Rossier School of Education University of Southern California) Retrieved from http://digitallibrary.usc.edu/assetserver/controller/item/ etd-McCord-3573.pdf;jsessionid=4D7B1E8084022B17D2F23EFFF2BF01D0

Mönks, F.J. y Pflüger, R. (2005). Gifted Education in 21 European Countries: Inventory and Perspective. Retrieved in December 2006 of www.bmbf.de/pub/gifted_education_21_eu_countries.pdf

Mönks, F.J. y Mason, E.J (2000).Developmental Psychology and Giftedness: Theories and Research. International Handbook of Giftedness and Talent (2 edition). (pp. 141-155). Oxford: Pergamon.

National Association for Gifted Children (1995). Standards for graduate programs in gifted education. Washington, DC: Author. Retrieved from http://www.nagc.org/index.aspx?id=394

National Quality Standards in Gifted and talented education (2005). QS Model. Retrieved from www.torbay.gov.uk/dfes-iqs-userguide.pdf

Penta UC (2008). Diseño módulos diplomado a distancia Psicología y Educación de Alumnos con Talentos Académicos. Documento no publicado.

Preckel, F., Goetz, T., Pekrun, R. y Kleine, M. (2008). Gender Differences in Gifted and AverageAbility Students: Comparing Girls' and Boys' Achievement, Self-Concept, Interest, and Motivation in Mathematics. Child Quarterly, 52(2), 146-159.

Redondo, J., Descouviéres, C. y Rojas, K. (2004). Equidad y Calidad de la Educación en Chile. Reflexiones e Investigaciones de Eficiencia de la Educación Obligatoria (1990-2001). Universidad de Chile: Santiago de Chile.

Renzulli, J. (1978). What makes giftedness? Re-examining a definition. Phi Delta Kappan, 60, 180-184, 261.

Rodríguez, M. (2006). La Atención a Niños con Facultades Talentosas Sobresalientes en el Marco de un Programa Estatal. Paper presented at the VI Congreso Iberoamericano De Superdotación, Talento y Creatividad, Fycomundit, Mar del Plata, Argentina. Retrieved from www.paenftsperu. com/conferencias/02sep_conf.doc

Rosner, W. (2009, Abril). Estándares de calidad en la formación de profesores de alumnos con altas capacidades. Retrieved from http://tvaustral.uach.cl/Play.aspx?video=A2D90D85592F50 A4DEDAD7C6702ADC15

Siegle, D. (2002). Creating a Living Portfolio: Documenting Student Growth With Electronic Portfolios, Gifted Child Today, 25(3), 60-63. 
Silverman, D. y Marvasti, A. (2008). Doing Qualitative Research. A Compressive Guide. USA: Sage Publications.

Sternberg, R. y Grigorenko, E. (2000). Teaching For Successful Intelligence. To Increase Student Learning and Achievement. Illinois: Arlington Heights.

Sternberg, R.J. y Subotnik, R.F. (2000). A Multidimensional Framework for Synthesizing Disparate Issues in Identifying Selecting, and Serving Gifted. Students. In Heller, K.A., Mönks, F.J., Sternberg, R.J. y Subotnik, R.F. (Eds.). International Handbook of Giftedness and Talent. (pp. 831-838). (2nd Edition). Kidlington, Oxford: Pergamon.

Tannenbaum, A.J. (2003). Nature and nurture of giftedness. In N. Colangelo y G. A. Davis (Eds.), Handbook of gifted education (3rd ed., pp. 45-59). Boston: Allyn y Bacon.

Tomlinson, C. (1999). The Differentiated Classroom. Responding the Needs of All Learners. Association for Supervision and Curriculum Development. VA USA: Alexandria.

Vantassel-Baska, J. y Johnsen, S. (2007). Teachers Education Standards for the Field of Gifted Education: A Vision of Coherence for Personnel Preparation in the 21st Century. Gifted Child Quarterly, 51, 182-205.

Van Tassel-Baska, J. (2002). Planning effective curriculum experiences for gifted learners. Understanding Our Gifted, 15(1), 6-8 Retrieved from http://www.davidsongifted.org/db/ Articles_id_10278.aspx

Van Tassel-Baska, J. (2000). Assessment of Gifted Student Learning in the Language Arts. The Journal of Secondary Gifted Education, 13 (2), 67-72.

Vialle, W. y Quigley, S. (2002). "The Teachers We Want”: Exploring the views of gifted students. En Mönks, F. y Wagner, H. (Ed.). Development of Human Potential: Investment into our future. Proceedings of the 8th Conference of the European Council for High Ability (ECHA) 125-128. Boon: Germany.

Winebrenner, S. (2003). Teaching Strategies for Twice-Exceptional Students. Intervention in School and Clinic, 38(3), 131-137.

Yoon, S. y Gentry, M. (2009). Racial and Ethnic Representation in Gifted Programs: Current Status of and Implications for Gifted Asian. Child Quarterly, 53 (2), 121-136. 
